\title{
Single-Event Effect Performance of a Conductive-Bridge Memory EEPROM
}

Dakai Chen ${ }^{1}$, Edward Wilcox ${ }^{2}$, Melanie Berg ${ }^{2}$, Hak Kim² ${ }^{2}$, Anthony Phan², Marco Figueiredo ${ }^{3}$, Christina Seidleck ${ }^{2}$ and Kenneth LaBel ${ }^{1}$,

1. NASA Goddard Space Flight Center, code 561, Greenbelt MD, 20771 2. ASRC Space and Defense, Greenbelt, MD, USA 20771 3. Orbital Science Corp., Greenbelt, MD, USA 20771

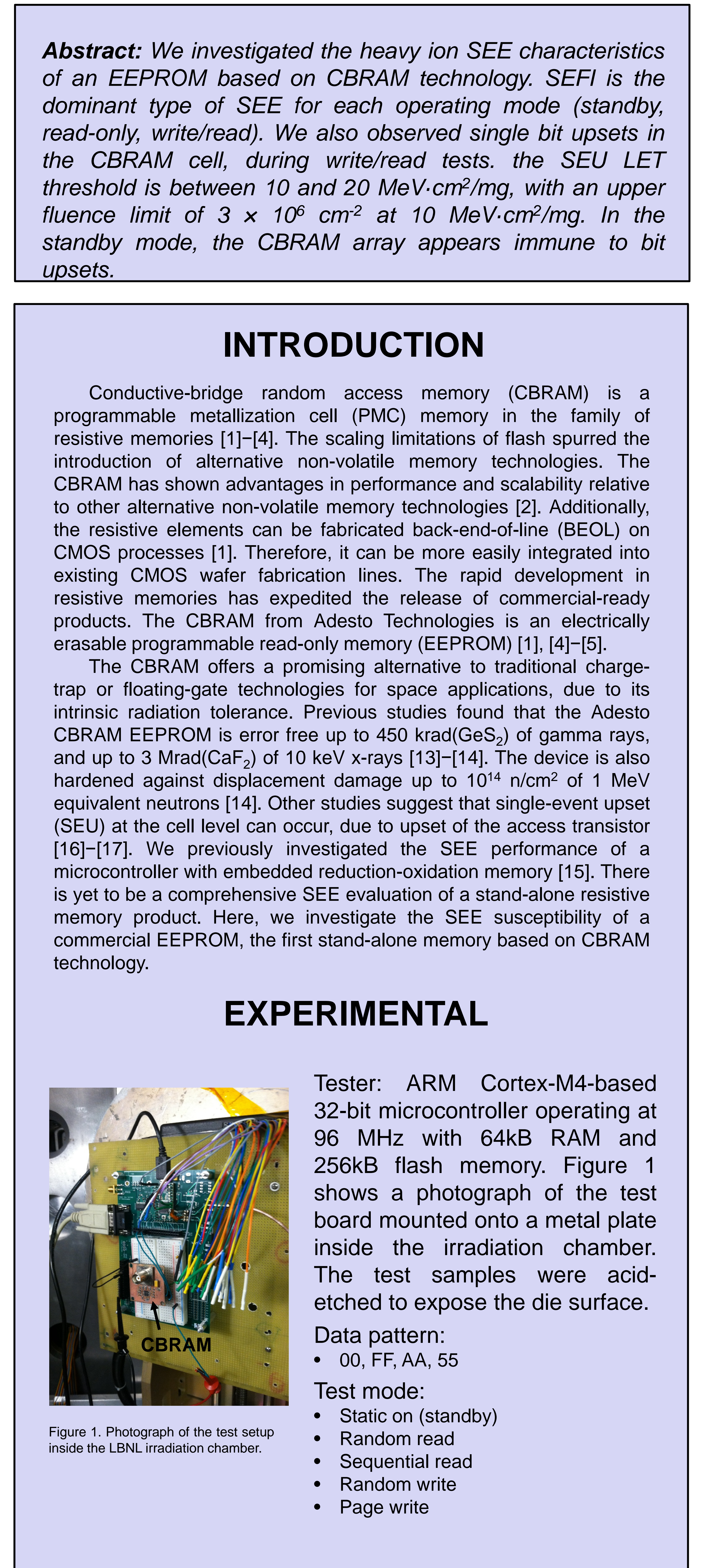

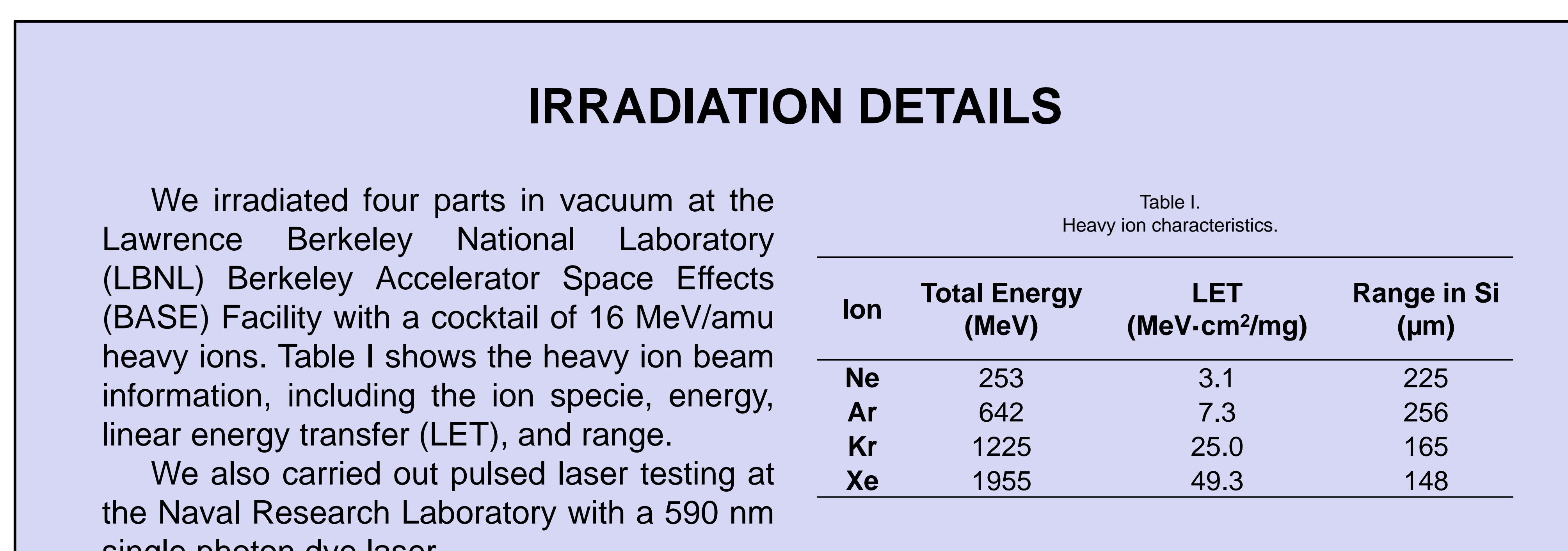

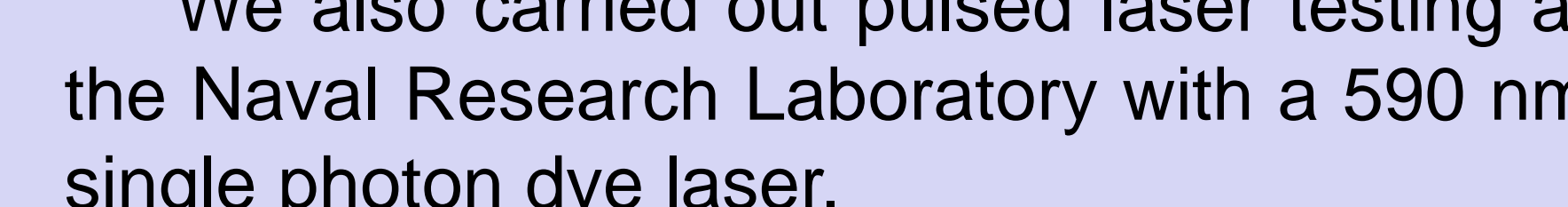

\section{DEVICE DETAILS}

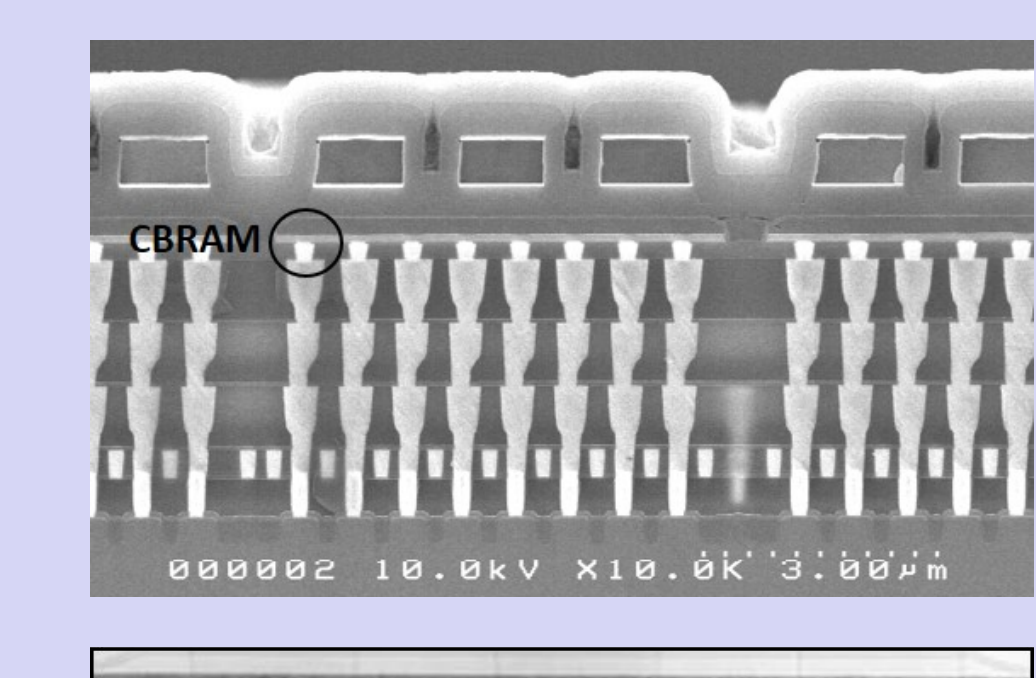

newion
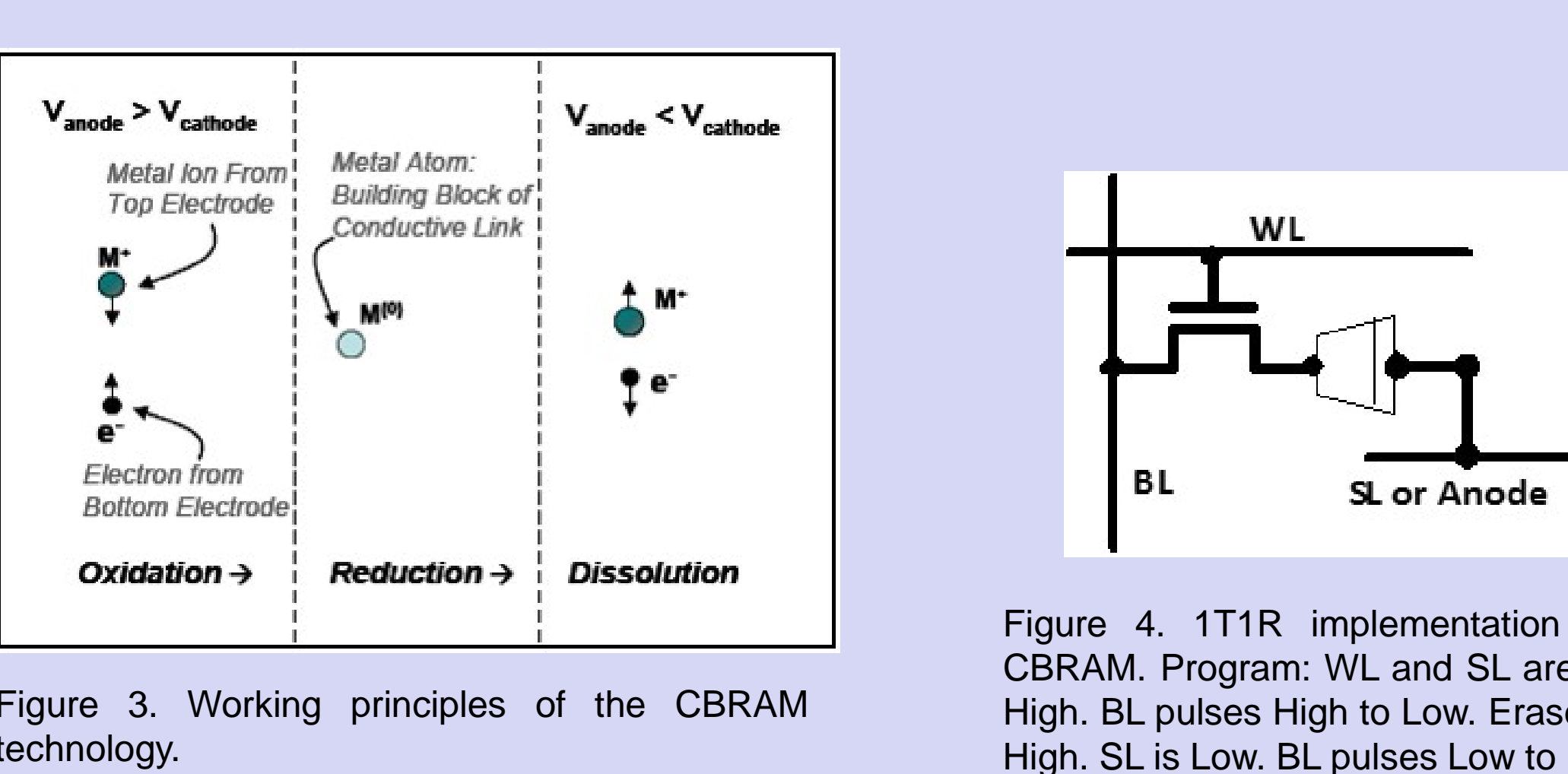

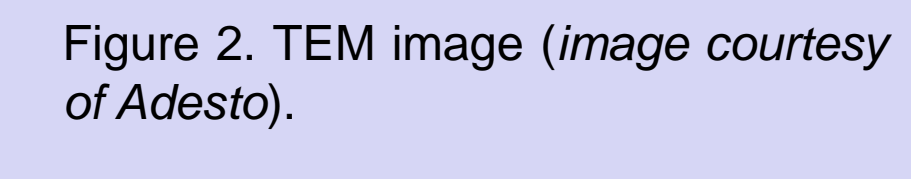

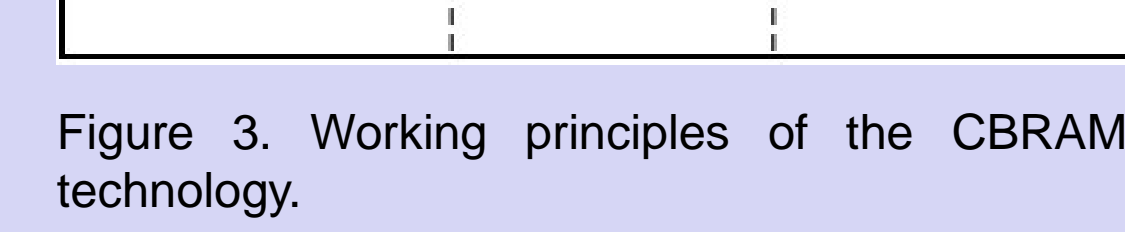

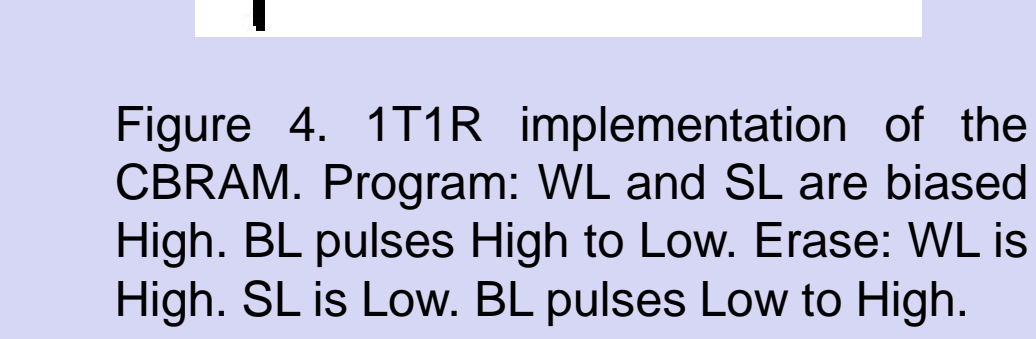

The RM24C from Adesto Technologies is the industry's first stand-alone EEPROM built with
CBRAM technology [1] [4]-[5] The EEPROM is available in 32,64 or $128 \mathrm{~Kb}$. Figure 3 shows a schematic diagram illustrating the physical mechanism of the CBRAM. Figure 4 shows a
schematic diagram of the one-transistor-one-resistor (1T1R) architecture of a CBRAM cell. TO program a cell, the Word Line (WL) and the anode or Select Line (SL) is High. The Bit Line (BL) pulses High to Low, which forward biases the CBRAM. To erase a cer
is low. The BL pulses Low to High, which reverse biases the CBRAM.

SINGLE EVENT UPSET

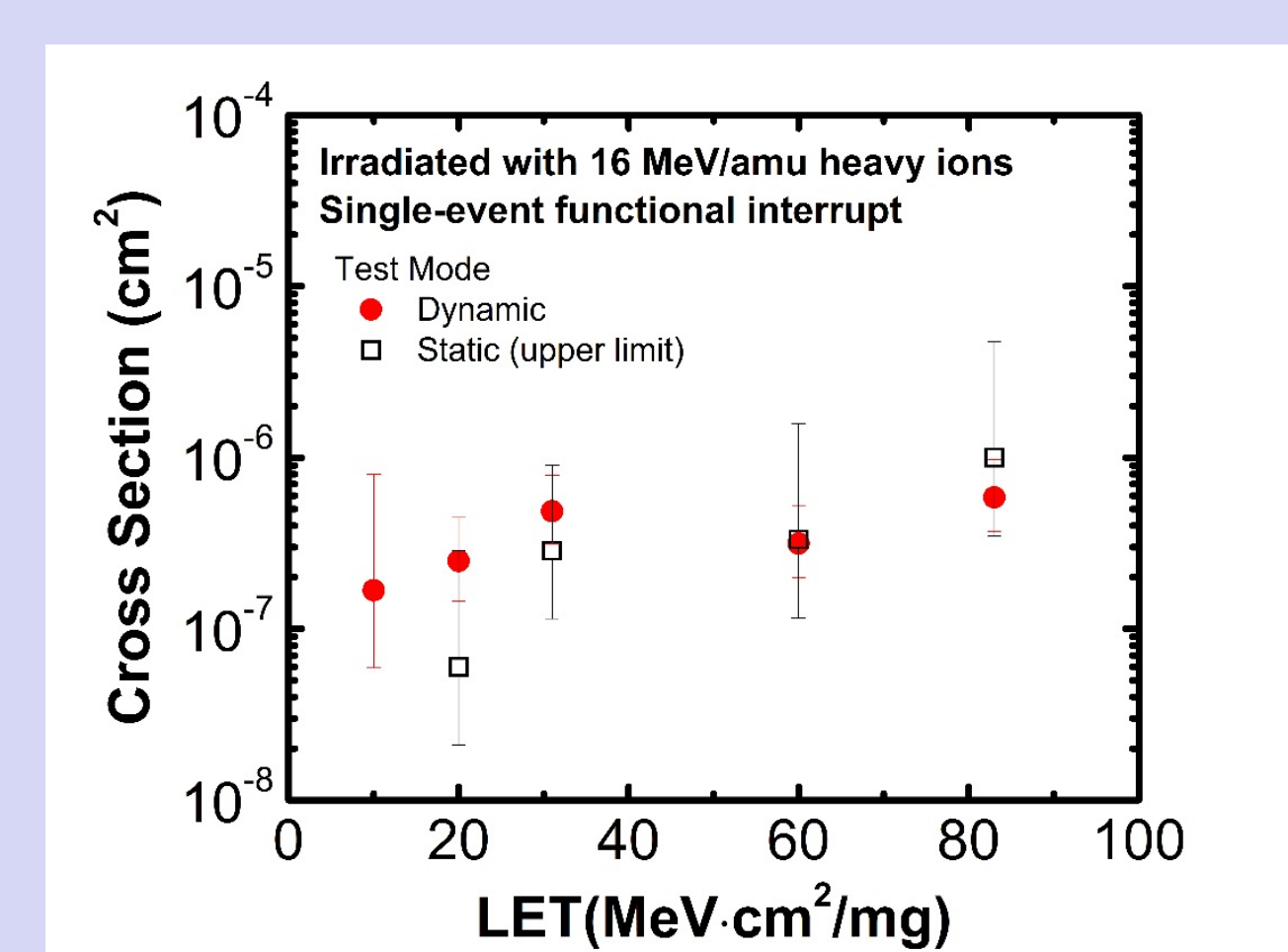

SBU characteristics:

: $\mathrm{LET}_{\mathrm{t}}$ between 10 and $20 \mathrm{MeV} \cdot \mathrm{cm} 2 \mathrm{mg}$
Observed in Write/Read mode only; immune during

static and read-only tests
Consisted of 1 to 0 and 0 to 1 type errors: athoug

1 to 0 errors are theoretically more likely for these
devices

devices
No angular dependence

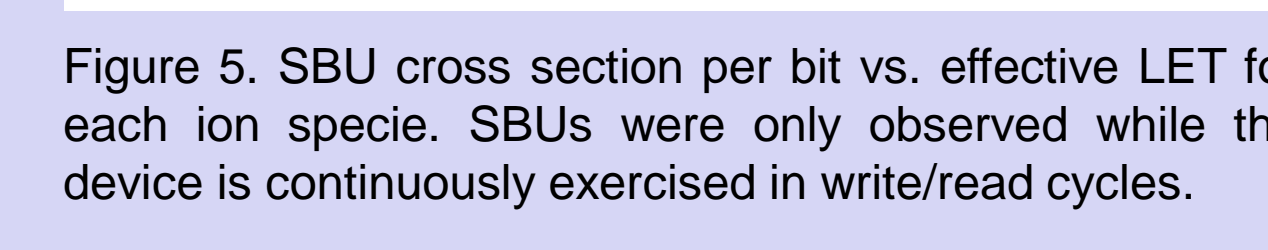
Figure 5 shows the single-bit upset cross section as a function of LET for each ion specie,
The relatively high error standard deviations are the result of the low count of the uppests. The
SBUS were rare relativive to SEUS that were characteristic of control circuit upsets. We fitered the errors which can be cleared by a second read (likely due to buffer upsets) and SEUs that cleared
atter power cycle (single-event transient (SEU) in the peripheral circuits).

SBU in the CBRAM cell is possible, consistent with previous studies on test structures [16], [17]
However there are key distinctions for the SBU observed here. For the standalone memory product here, the most vulnerable state for the access transistor is during erase rather than program. The transistors
with reverse biased drain are located in the same row as the target cell to be erased. As a result, a SEU of the opposite polarity will be less likely. However, the fact that we see errors of both polartitis raise
questions about the origin of the SEUs. It is possible that some of these SEUs are due to unidentified buffer uspets. A larger sample size is needed to investigate the SEU characteristics further. Nonetheless, SINGLE EVENT FUNCTIONAL INTERRUPT

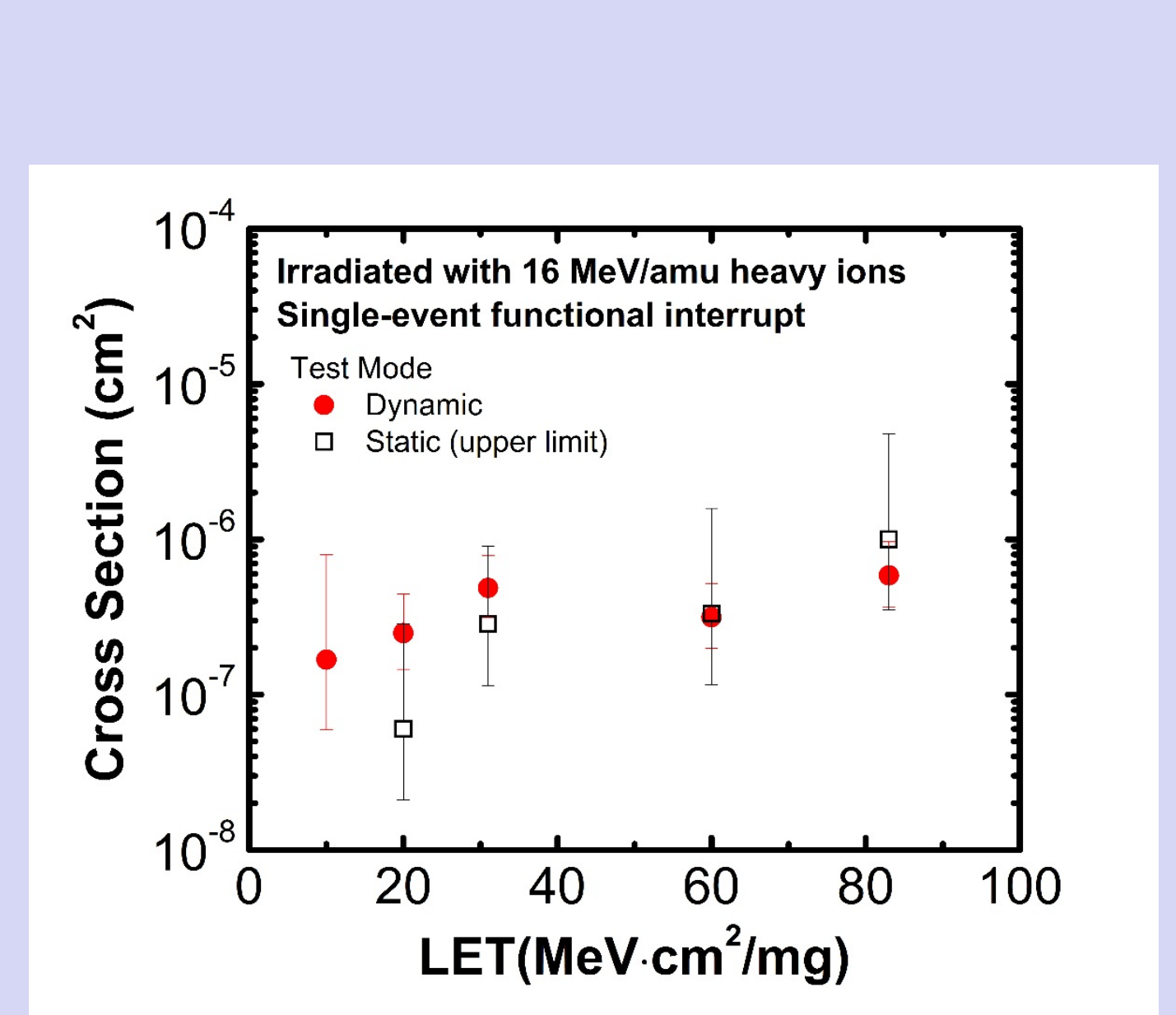

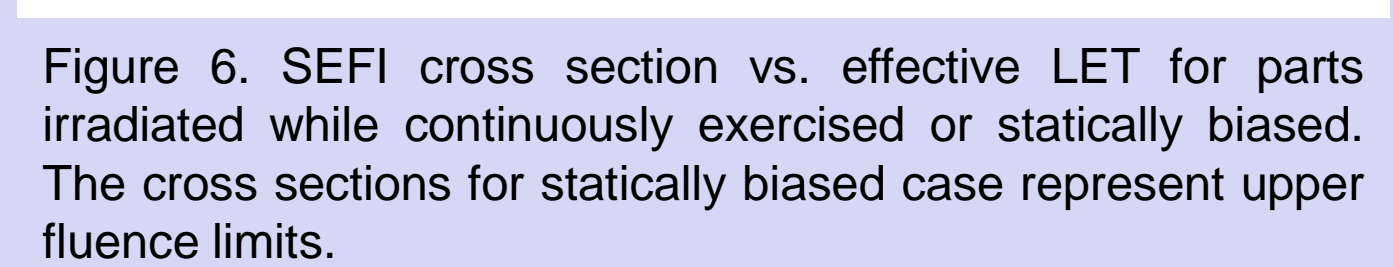

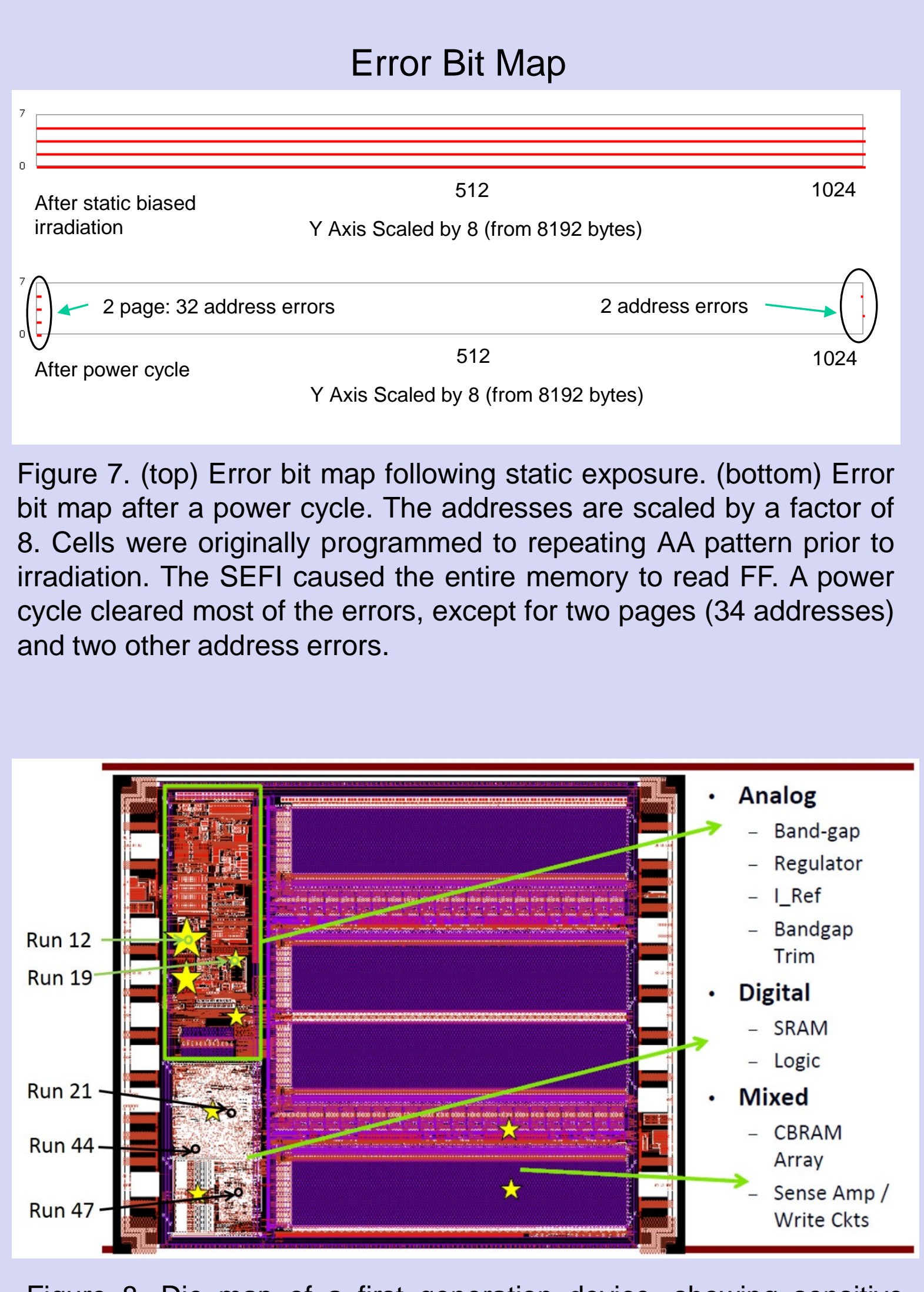

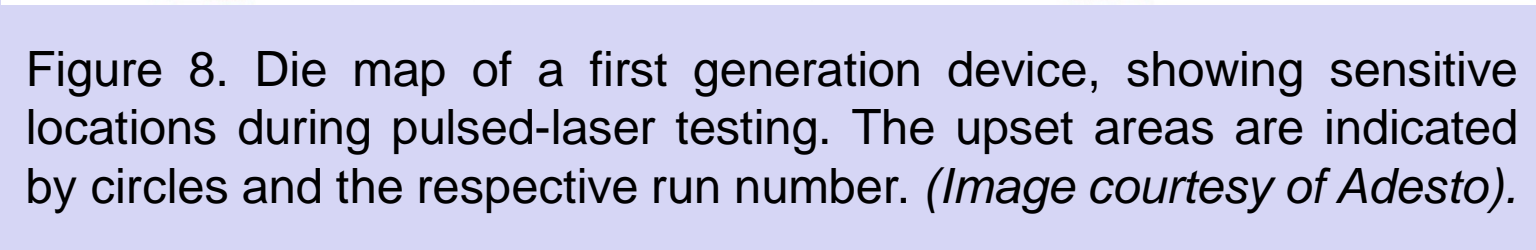

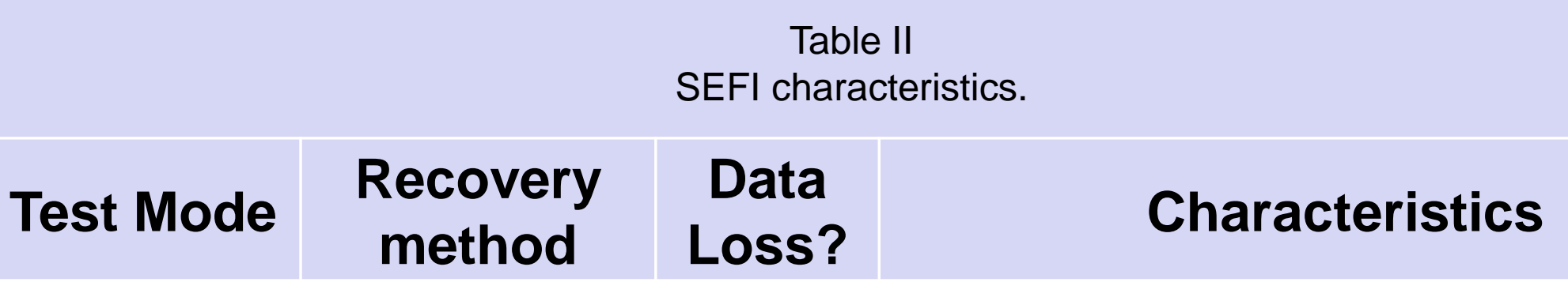

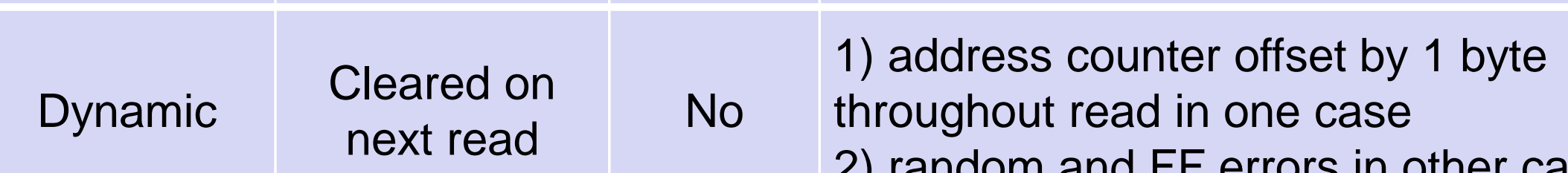

$$
\begin{aligned}
& \begin{array}{c}
\text { Static and } \\
\text { Dynamic }
\end{array}
\end{aligned}
$$

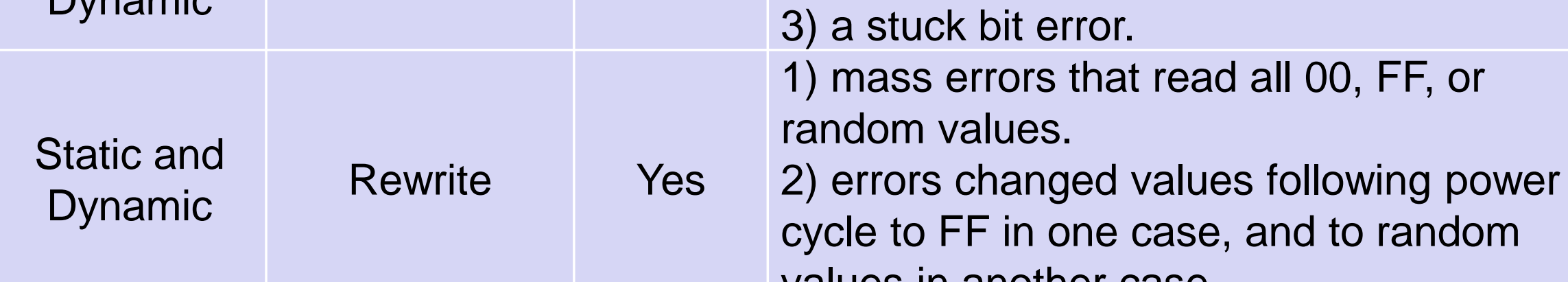

Figure 6 shows the SEFI cross section vs. effective LET
for static and dynamic test modes. Table II categorizes the for static and dynamic test modes. Table II categorizes the
SEFIS. A potentially dangerous SEFF occurred during static

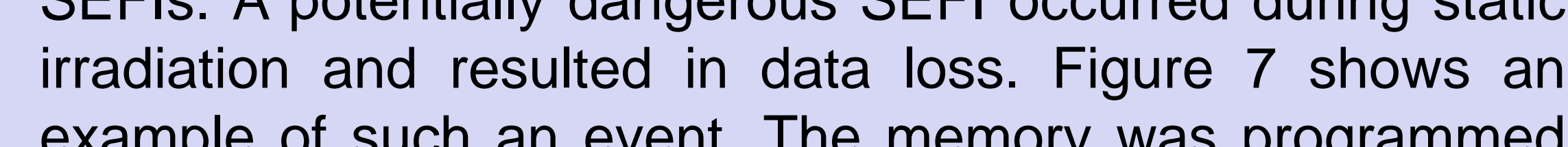
example of such an event. The memory was programmed
with a repeating AA pattern. After the irradiation, all of the memory space read $F F$ (top). A power cycle cleared most of the errors, except for two pages and two SEUS (bottom).
These errors signify data corruption during the beam exposure or during read-back. These errors could only be cleared by rewriting the cells. This type of SEFI often pages. Furtrenermore, in some cases, the supply current
showed spikes un to approximately $2-3 \mathrm{~mA}$ during

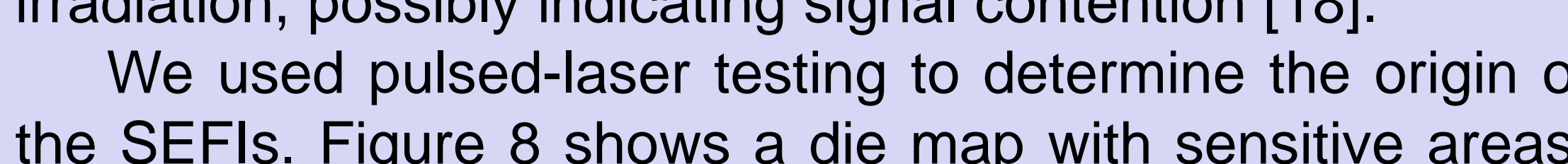
We determined that SEFI can be triggered from strikes on Memory (SRAM), and logic circuits. Strikes on the sense amplifier circuits did not lead to upsets, contrary to a
previous investigation on another resistive memory device [15]. The metallization overlayers and the residue on the
surface of the die likely obstructed the pulsed laser from fully penetrating these sensitive regions. Nonetheless, the pulsed-laser test showed that the CRRAM array Is
robust against ionizing radiation-induced upsets.
CONCLUSION

The CBRAM appears to be hardened against heavy ion-
induced bit upset when powered off or in standby mode. unlike floating-gate or trap-charge flash and EEPROMS. The
SEF I response is similar to existin EEPROM and flash
lechnologpies, with emiror modedes that include page erlors, mass memory errors, device hang-ups, etc. However, unlike
modern flash devices, the CBRAM show similar SEFI

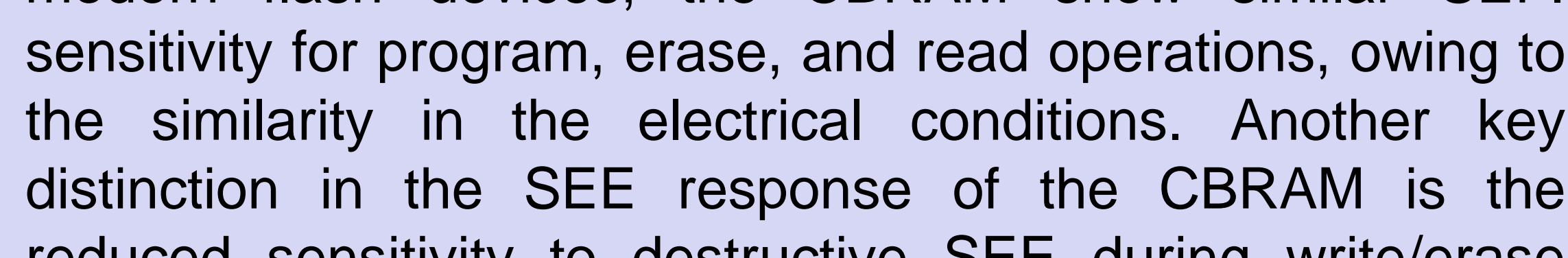
reduced sensitivity to destructive SEE during write/larase
operations. The vulnerability to SEDR is reduced

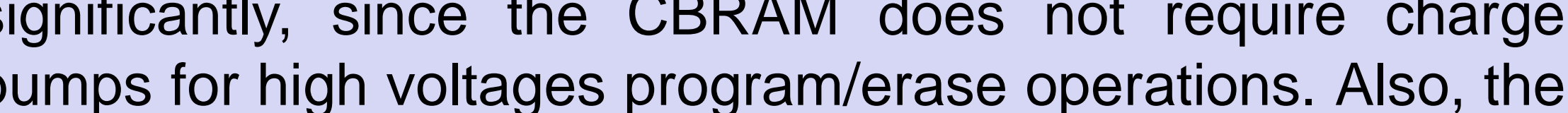
pumps tor high voltages program/erase operations. Asso, the
fact that the CBAM is fabricated BEOL on a standard CMOS process suggests it could be developed into a space-
grade product. The manutacturer or other appopopiate
militaryspace chip manufacturers can potentially transfer the CBRAM technology onto a radiation-hardened plattorm without a complete redesign of the fabrication process. This
distiction offers another advantage for the CBRAM's
potential utilization for space missions. a high density CBRAM here. As the technology progresses, the sizz of the CBRAM stack will shrink and the cell-to-cell noise margin will
decrease, both aspects could impact the radiation tolerance.

ACKNOWLEDGEMENT

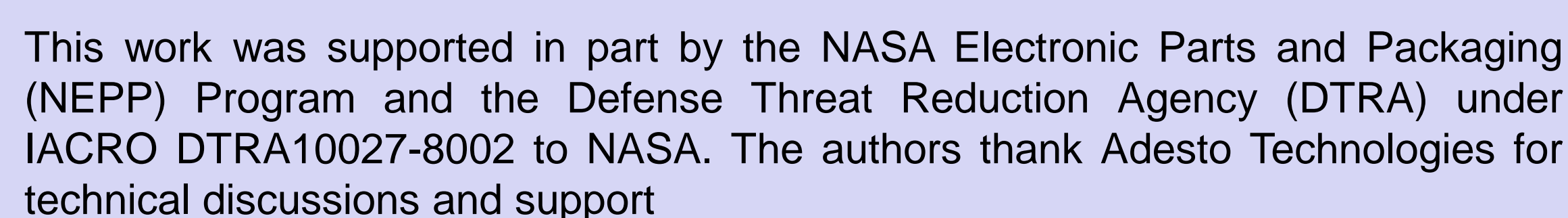
REFERENCE

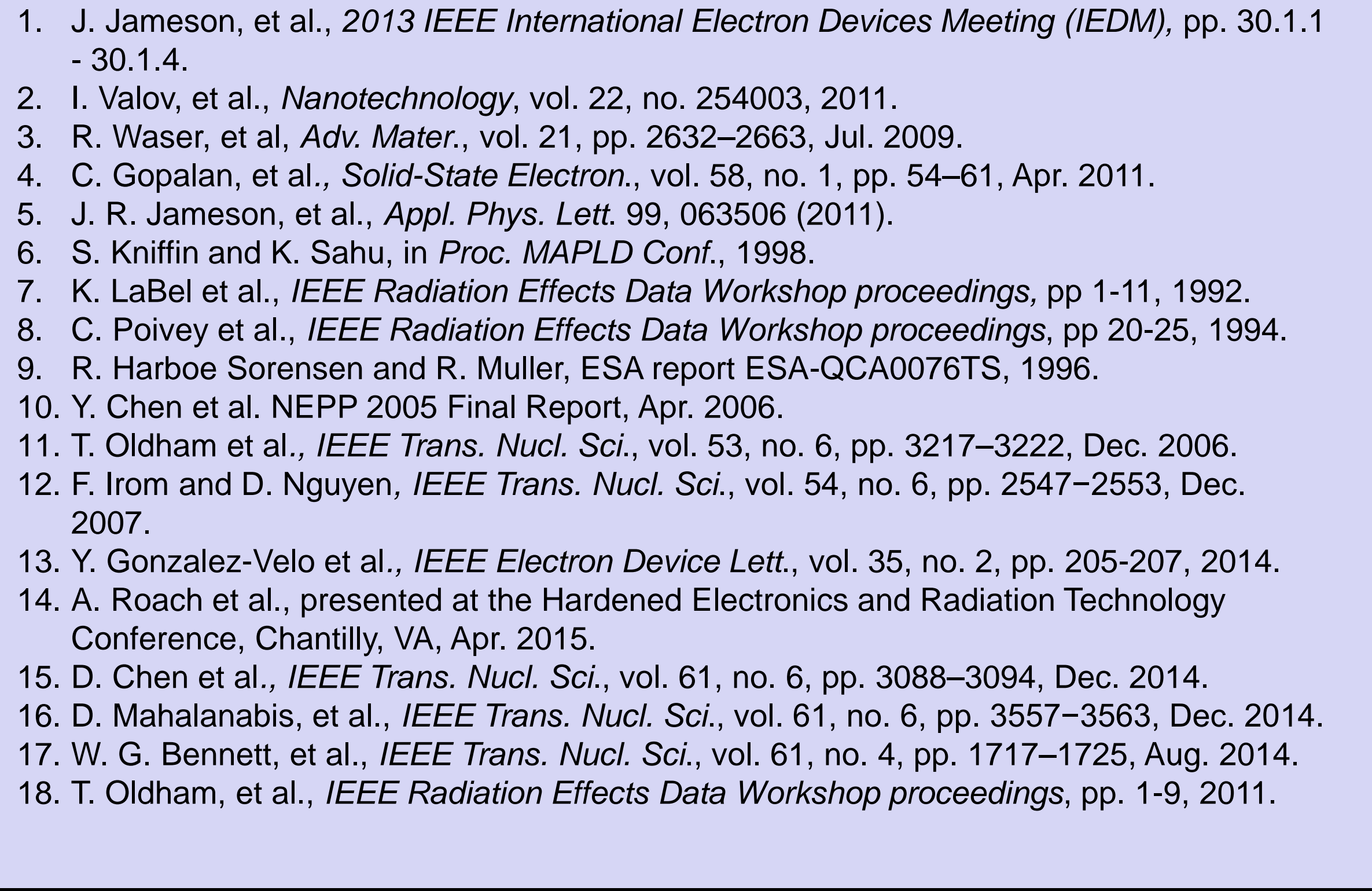

